\title{
Atmospheric Circulations of Hot Jupiters as Planetary Heat Engines
}

\author{
Daniel D. B. Koll ${ }^{1,3}$ (iD and Thaddeus D. Komacek ${ }^{2,3}$ (iD \\ ${ }^{1}$ Department of Earth, Atmospheric, and Planetary Sciences, Massachusetts Institute of Technology, Cambridge, MA 02139, USA; dkoll@mit.edu \\ ${ }^{2}$ Lunar and Planetary Laboratory and Department of Planetary Sciences, University of Arizona, Tucson, AZ 85721, USA; tkomacek@1pl.arizona.edu \\ Received 2017 October 6; revised 2017 December 7; accepted 2017 December 19; published 2018 January 31
}

\begin{abstract}
Because of their intense incident stellar irradiation and likely tidally locked spin states, hot Jupiters are expected to have wind speeds that approach or exceed the speed of sound. In this work, we develop a theory to explain the magnitude of these winds. We model hot Jupiters as planetary heat engines and show that hot Jupiters are always less efficient than an ideal Carnot engine. Next, we demonstrate that our predicted wind speeds match those from three-dimensional numerical simulations over a broad range of parameters. Finally, we use our theory to evaluate how well different drag mechanisms can match the wind speeds observed with Doppler spectroscopy for HD $189733 \mathrm{~b}$ and HD 209458b. We find that magnetic drag is potentially too weak to match the observations for HD 189733b, but is compatible with the observations for HD 209458b. In contrast, shear instabilities and/or shocks are compatible with both observations. Furthermore, the two mechanisms predict different wind speed trends for hotter and colder planets than currently observed. As a result, we propose that a wider range of Doppler observations could reveal multiple drag mechanisms at play across different hot Jupiters.
\end{abstract}

Key words: hydrodynamics - methods: analytical - methods: numerical - planets and satellites: atmospheres planets and satellites: individual (HD 189733b, HD 209458b)

\section{Introduction}

Hot Jupiters provide a unique laboratory for testing our understanding of planetary atmospheres. Showman \& Guillot (2002) were the first to consider the atmospheric circulations of these planets. Using numerical simulations, Showman \& Guillot predicted that hot Jupiters should develop strongly superrotating equatorial jets, with wind speeds up to several kilometers per second. This prediction was confirmed by subsequent observations, which showed that the thermal emission peak on many hot Jupiters is shifted eastward from the substellar point, consistent with heat being advected downwind by a superrotating jet (e.g., Knutson et al. 2007; Crossfield et al. 2010).

More recent observations have started to directly constrain the wind speeds of these jets. High-resolution transmission spectra have found Doppler shifts in molecular absorption lines for HD 209458b (Snellen et al. 2010) as well as HD 189733b (Louden \& Wheatley 2015; Wyttenbach et al. 2015; Brogi et al. 2016). The significant $\left(\sim\right.$ several $\left.\mathrm{km} \mathrm{s}^{-1}\right)$ blueshifts detected for both planets imply rapid dayside-to-nightside winds that are broadly consistent with the wind speeds predicted by a range of numerical simulations (Showman \& Guillot 2002; Showman et al. 2009; Heng et al. 2011a; Showman et al. 2013; Komacek et al. 2017).

Although it is qualitatively understood why hot Jupiters develop equatorial jets, there is still no general theory that explains the jets' magnitude. Hot Jupiters are very likely tidally locked. This orbital spin state creates a strong day-night forcing which excites standing waves that flux angular momentum toward the equator and drive equatorial superrotation (Showman \& Polvani 2011). The strength of superrotation should therefore depend on the ratio between horizontal wave propagation and radiative cooling timescales (Koll \& Abbot 2015; Komacek \& Showman 2016; Zhang \&

\footnotetext{
${ }^{3}$ The authors contributed equally to this work, and are listed alphabetically.
}

Showman 2017). This basic expectation is complicated, however, by results that show that the jet's state depends on both horizontal standing waves and vertical eddies (Tsai et al. 2014; Showman et al. 2015), and it is still unclear how the two mechanisms jointly determine the jet's magnitude.

In this paper, we constrain the wind speeds of hot Jupiters by modeling their atmospheric circulations as planetary heat engines. The utility of this approach has previously been demonstrated for hurricanes on Earth (Emanuel 1986) and rocky exoplanets (Koll \& Abbot 2016). Atmospheric circulations can be considered as heat engines because parcels of fluid tend to absorb heat at a high temperature (e.g., on the dayside of a hot Jupiter) and emit heat at a low temperature (on the nightside). The differential heating and cooling allows parcels to generate work, and thus kinetic energy, which in steady state has to be balanced by the dissipation of kinetic energy via friction.

In contrast to hurricanes and the atmospheres of rocky exoplanets, however, it is still poorly understood how hot Jupiters dissipate kinetic energy (Goodman 2009). Potential mechanisms include magnetic drag in partially ionized atmospheres (Perna et al. 2010; Menou 2012; Rauscher \& Menou 2013; Rogers \& Showman 2014), shocks in supersonic flows (Li \& Goodman 2010; Heng 2012; Perna et al. 2012; Dobbs-Dixon \& Agol 2013; Fromang et al. 2016), and turbulence induced by fluid instabilities such as the KelvinHelmholtz instability (Li \& Goodman 2010; Fromang et al. 2016).

Our goal is to evaluate these proposed mechanisms and to test which of them are able to match current observations. To do so, we first describe our numerical simulations (Section 2). Next, we develop the heat engine framework and test it with the numerical simulations (Section 3). Finally, we apply our framework to observations (Section 4) and state our conclusions (Section 5). Our results show that current observations favor shear instabilities and/or shocks as the dominant drag 
mechanism for HD 189733b, and motivate extending similar observations across a wider range of planets.

\section{Numerical Simulations}

We compare our theory with the GCM simulations that were previously described in Komacek et al. (2017). In summary, the simulations use the MITgcm (Adcroft et al. 2004) to solve the atmospheric fluid dynamics equations coupled to double-gray radiative transfer with planetary parameters relevant for a typical hot Jupiter, HD 209458b. The double-gray approximation divides the spectrum into an incoming collimated and a thermal diffuse part. The absorption coefficients were chosen to match more detailed radiative transfer calculations; the absorption coefficient for incoming stellar radiation is set to a uniform value, $\kappa_{\mathrm{SW}}=4 \times 10^{-4} \mathrm{~m}^{-2} \mathrm{~kg}^{-1}$, the thermal absorption coefficient varies approximately with the square root of pressure, $\kappa_{\mathrm{LW}}=2.28 \times 10^{-6} \mathrm{~m}^{-2} \mathrm{~kg}^{-1} \times(p / 1 \mathrm{~Pa})^{0.53}$, where the power-law exponent comes from fitting the analytic model of Parmentier \& Guillot (2014) and Parmentier et al. (2015) to radiative transfer models with realistic opacities. With these values, the photosphere (where the optical thickness equals unity) for stellar radiation lies at about 0.23 bar and the photosphere for thermal radiation lies at 0.28 bar.

The model's resolution is C32 in the horizontal (roughly corresponding to a global resolution of $128 \times 64$ in longitude and latitude) and 40 levels in the vertical, which are evenly spaced in log pressure, with the uppermost layer extending to zero pressure. Table 1 summarizes the physical and numerical parameters used in our suite of models.

Most GCMs do not explicitly resolve the mechanisms that are thought to dissipate kinetic energy in hot-Jupiter atmospheres, such as Lorentz drag or shocks (see Section 1). Our GCM includes two potential sources of drag, which can be thought of as parametrizing these mechanisms. First, the simulations include a Rayleigh drag that linearly damps winds

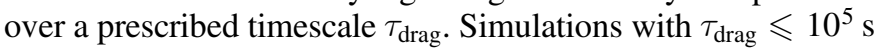
use a timescale that is spatially uniform. Simulations with $\tau_{\text {drag }}>10^{5} \mathrm{~s}$ additionally include a "basal" drag term that allows the model to equilibrate within reasonable integration times. The basal drag strength increases as a power law with pressure, from no drag at 10 bar to a timescale of 10 days at 200 bar (Komacek \& Showman 2016). Second, to enforce numerical stability, the model includes a fourth-order Shapiro filter that damps wind and temperature variations at the model grid scale. The Shapiro filter acts as numerical drag at small spatial scales and, in simulations without any other sources of drag, eventually helps to equilibrate the kinetic energy of the flow. The potential issue with relying on numerical drag is that it relies on parameters that are generally chosen for modeling convenience, not because they are physically motivated. This raises the question of which source of drag is dominant in our simulations.

We find that numerical drag can play a key role in our GCM simulations. Although the potential importance of numerical drag has repeatedly been pointed out in the hot-Jupiter literature (Goodman 2009; Li \& Goodman 2010; Thrastarson \& Cho 2010; Heng et al. 2011a; Liu \& Showman 2013; Mayne et al. 2014; Polichtchouk et al. 2014; Cho et al. 2015), no work has previously quantified its effect relative to explicitly parametrized drag. Figure 1 compares the rates at which our GCM is dissipating kinetic energy via numerical drag from the Shapiro filter versus the dissipation rate due to Rayleigh drag as
Table 1

Range of Physical and Numerical Parameters Used in Our Suite of Simulations

\begin{tabular}{|c|c|c|}
\hline Physical Parameter & Parameter Value(s) & Unit \\
\hline $\begin{array}{l}\text { Equilibrium temperature } \\
\qquad T_{\text {eq }}\end{array}$ & $\begin{array}{c}500,1000, \mathbf{1 5 0 0}, 2000 \\
2500,3000\end{array}$ & $\mathrm{~K}$ \\
\hline $\begin{array}{l}\text { Visible absorption } \\
\text { coefficient } \kappa_{\mathrm{SW}}\end{array}$ & $4 \times 10^{-4}$ & $\mathrm{~m}^{-2} \mathrm{~kg}^{-1}$ \\
\hline $\begin{array}{c}\text { Thermal absorption } \\
\text { coefficient } \kappa_{\mathrm{LW}}\end{array}$ & $2.28 \times 10^{-6} \times(p / 1 \mathrm{~Pa})^{0.53}$ & $\mathrm{~m}^{-2} \mathrm{~kg}^{-1}$ \\
\hline Drag timescale $\tau_{\text {drag }}$ & $10^{3}, 10^{4}, 10^{5}, 10^{6}, 10^{7}, \infty$ & $\mathrm{s}$ \\
\hline Gravity $g$ & 9.36 & $\mathrm{~m} \mathrm{~s}^{-2}$ \\
\hline Rotation rate $\Omega$ & $2.078 \times 10^{-5}$ & $\mathrm{~s}^{-1}$ \\
\hline Planet radius $a$ & $9.43 \times 10^{7}$ & $\mathrm{~m}$ \\
\hline Heat capacity $C_{p}$ & $1.3 \times 10^{4}$ & $\mathrm{~J} \mathrm{~kg}^{-1} \mathrm{~K}^{-1}$ \\
\hline Specific gas constant $R$ & 3700 & $\mathrm{~J} \mathrm{~kg}^{-1} \mathrm{~K}^{-1}$ \\
\hline Numerical parameter & Parameter Value(s) & Unit \\
\hline Horizontal resolution $\left(N_{x}\right)$ & C16(64), C32 (128), C64 (256) & $\mathrm{n} / \mathrm{a}$ \\
\hline Vertical resolution $N_{z}$ & 40 & $\mathrm{n} / \mathrm{a}$ \\
\hline Timestep $d t$ & $1.5,7.5,15$ & $\mathrm{~s}$ \\
\hline $\begin{array}{l}\text { Shapiro filter timescale } \\
\qquad d t_{\text {num }}\end{array}$ & $1.5,7.5,15,25$ & $\mathrm{~s}$ \\
\hline $\begin{array}{l}\text { Shapiro filter length } \\
\text { scale } l_{\text {num }}=2 \pi a / N_{x}\end{array}$ & $2 \pi a / 64, \mathbf{2} \pi \mathbf{a} / \mathbf{1 2 8}, 2 \pi a / 256$ & $\mathrm{~m}$ \\
\hline Shapiro filter order $n$ & 4 & $\mathrm{n} / \mathrm{a}$ \\
\hline
\end{tabular}

Note. Numerical parameters in bold show fiducial values used for our main suite of simulations with varying physical parameters, and physical parameters in bold highlight fiducial values used for our secondary suite of simulations with varying numerical parameters. Numbers in parentheses for horizontal resolution show the approximate number of horizontal grid points.

a function of pressure. Figure 1(a) shows the relative global root-mean-square dissipation due to numerical drag versus Rayleigh drag, while Figure 1(b) shows the absolute global root-mean-square value of kinetic energy dissipated by both drag mechanisms. We compute the root-mean-square change in kinetic energy as $(\partial K / \partial t)_{\mathrm{rms}}=\left\langle(\partial K / \partial t)^{2}\right\rangle^{1 / 2}$, where the angle brackets denote an area average. We find that all simulations with moderately long Rayleigh drag timescales, $\tau_{\text {drag }} \geqslant 10^{6} \mathrm{~s}$, dissipate most kinetic energy through numerical drag.

Moreover, even in the simulations with the strongest Rayleigh drag (yellow curve in Figures 1(a), (b)) numerical drag dominates the dissipation of kinetic energy near the top and bottom of the model domain. Although the model includes a basal drag, we find that it contributes less toward kinetic energy dissipation than numerical drag near the bottom of the domain. This is likely due to the Shapiro filter acting as a sponge for waves that are excited in the upper atmosphere. However, wind speeds at pressures greater than 10 bar are small, so kinetic energy dissipation near the domain bottom contributes relatively little to the overall dissipation (see Figure 1(b)). 

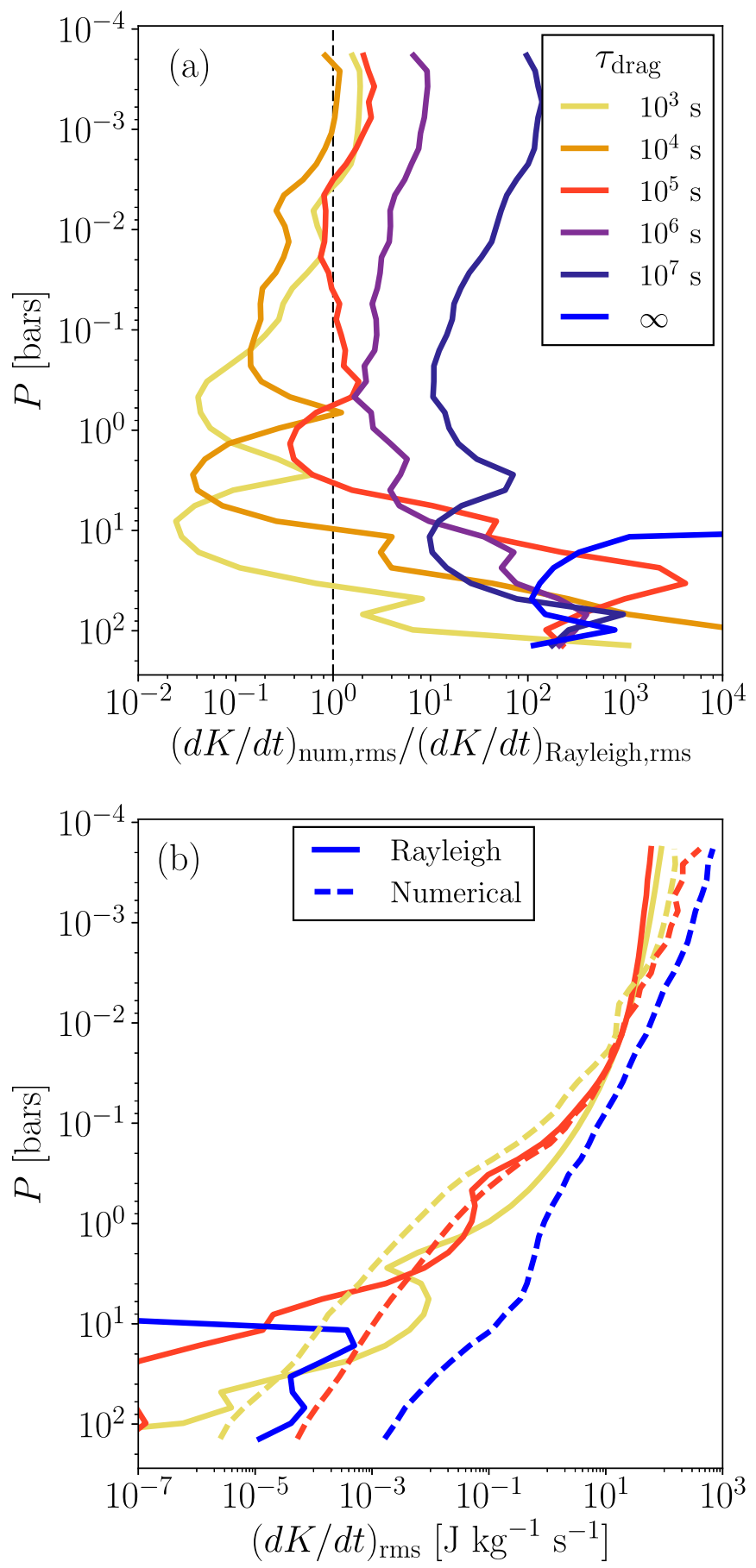

Figure 1. Kinetic energy dissipation in many of our GCM simulations is dominated by numerical drag. Panel (a) shows the ratio between the global root-mean-square rate of kinetic energy dissipation by numerical drag, $(d K / d t)_{\text {num,rms, }}$ vs. the global root-mean-square rate of kinetic energy dissipation by explicit Rayleigh drag, $(d K / d t)_{\text {Rayleigh,rms, }}$ as a function of pressure for models with an equilibrium temperature of $T_{\mathrm{eq}}=1500 \mathrm{~K}$. The colored lines show simulations with different Rayleigh drag timescales, with darker lines representing longer drag timescales. The dashed vertical line shows the divide between dissipation dominated by numerical drag (to the right of the line) and Rayleigh drag (to the left). Except for short Rayleigh drag timescales, $\tau_{\text {drag }} \leqslant 10^{4} \mathrm{~s}$, numerical dissipation dominates. Note that the case with $\tau_{\text {drag }}=\infty$ still includes basal drag, so the ratio of numerical to Rayleigh drag dissipation is not infinite at depth. Panel (b) shows the absolute contribution of Rayleigh drag and numerical effects to the kinetic energy dissipation. Only a subset of the simulations are shown for visual convenience. The dissipation rate increases with decreasing pressure, largely due to the stronger wind speeds at lower pressures.

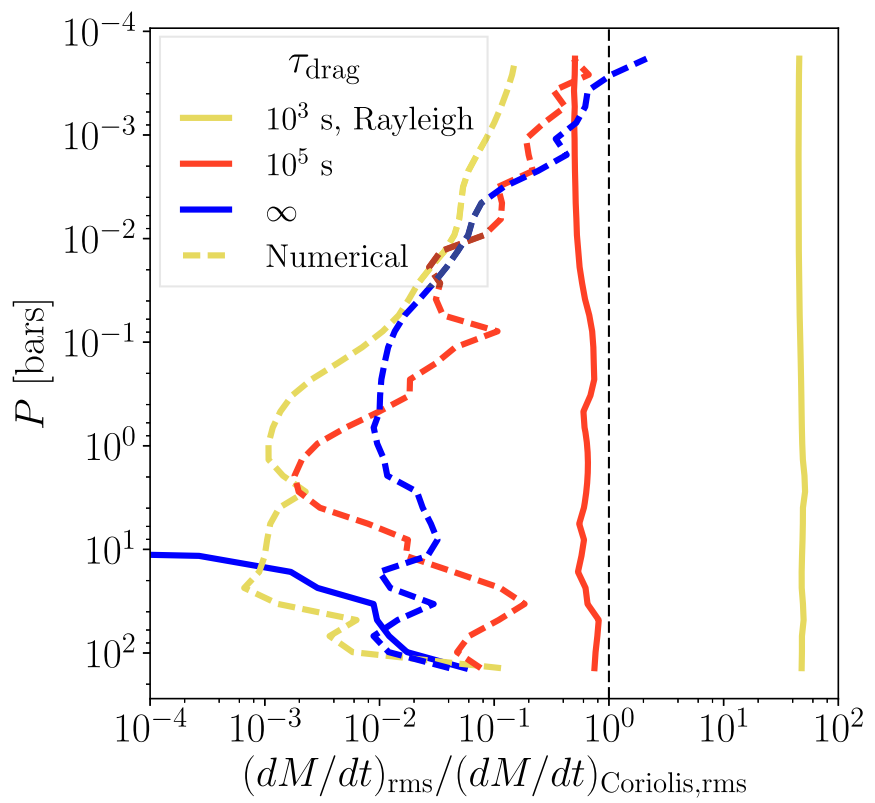

Figure 2. Numerical effects are small relative to physical terms in the zonal angular momentum budget of our simulations. This plot shows the global rootmean-square of the change in zonal momentum due to Rayleigh drag (solid lines) and numerics (dashed lines) relative to the change in zonal angular momentum due to the Coriolis force (i.e., rotation). Plots have the same color scheme as in Figure 1. For visual convenience, we only show a subset of all simulations. The acceleration from numerics is smaller than either the Coriolis force (if $\tau_{\text {drag }} \geqslant 10^{5} \mathrm{~s}$ ) or Rayleigh drag (if $\tau_{\text {drag }} \leqslant 10^{4} \mathrm{~s}$ ). As a result, numerics do not significantly affect the angular momentum budget of our simulations.

Though numerical drag is a dominant factor in how our GCM dissipates kinetic energy, atmospheric circulations additionally depend on how the GCM resolves the angular momentum budget. We do not expect a priori that numerical effects will dominate the global angular momentum budget, because the Shapiro filter is designed to not affect large-scale flow (Shapiro 1971). To check this insight, we explicitly compute the change in zonal angular momentum by numerics and Rayleigh drag as in Peixoto \& Oort (1992):

$$
\frac{\partial M}{\partial t}=\frac{\partial u}{\partial t} a \cos (\phi)
$$

In Equation (1), $M$ is the zonal angular momentum per unit mass, $\partial M / \partial t$ is the rate of change of angular momentum, which we compute in our simulations from the acceleration $\partial u / \partial t$ due to the Shapiro filter or Rayleigh drag, $a$ is the planetary radius, and $\phi$ is latitude. Rayleigh drag always acts as a sink of angular momentum whereas the Shapiro filter can accelerate parts of the flow so we compare both terms via the root-mean-square change in momentum, $(\partial M / \partial t)_{\mathrm{rms}}=\left\langle(\partial M / \partial t)^{2}\right\rangle^{1 / 2}$, where the angle brackets as before denote an area average.

We find that numerical effects play a relatively minor role in the zonal angular momentum budget. Figure 2 shows the change in angular momentum from numerics and Rayleigh drag relative to the change in angular momentum from the Coriolis force, as a function of pressure. We compare both terms against the Coriolis force because it is a small term in the zonal momentum budget of hot Jupiters due to their slow rotation and winds that peak at the equator (Showman \& Polvani 2011; Showman et al. 2015). In relative terms, we find that the numerical change in angular momentum becomes 
larger than Rayleigh drag once $\tau_{\text {drag }}>10^{5} \mathrm{~s}$ (blue curves). However, in absolute terms, the momentum change from numerics remains one to two orders of magnitude smaller than the Coriolis term at most pressure levels. We conclude that numerical effects likely do not play a dominant role in the angular momentum budget of our simulations.

Given that many published simulations of hot Jupiters do not include Rayleigh drag, our results indicate that many of these simulations rely on numerical drag to equilibrate kinetic energy. Further work is needed to ensure that this kind of dissipation in hot-Jupiter GCMs is physically motivated and that its effects are robust with respect to changes in numerical parameters. At the same time, the angular momentum budget in our simulations is not dominated by numerics. We therefore expect that GCMs are robust in simulating the qualitative features of hot-Jupiter circulations (e.g., equatorial jets), but that the absolute kinetic energy and thus wind speeds in these simulations might be affected by numerical details. Our results agree with previous work, which has shown that the equilibrated flows in hot-Jupiter GCMs largely conserve angular momentum, are independent of initial conditions, and the magnitude of winds is only weakly sensitive to changes in numerical parameters (e.g., Heng et al. 2011b; Liu \& Showman 2013; Mayne et al. 2014). In the remainder of this paper, we focus on existing GCMs to test our theoretical framework. To do so, we develop a theory in the next section that can account for both explicit and numerical drag.

\section{Hot Jupiters as Heat Engines}

In steady state, the rate $W$ at which a heat engine performs work is given by

$$
W=\eta Q,
$$

where $\eta$ is the engine's thermodynamic efficiency and $Q$ is the rate at which the engine absorbs heat.

First, the heating rate $Q$ is equal to the average absorbed stellar flux,

$$
Q=\sigma T_{\mathrm{eq}}^{4},
$$

where $T_{\mathrm{eq}}$ is the planetary equilibrium temperature.

Second, we constrain the work output rate $W$. We assume that work goes entirely toward generating and dissipating kinetic energy. If Rayleigh drag dominates, the rate at which kinetic energy is dissipated equals

$$
W_{\text {Rayleigh }}=\int \frac{d p}{g} \times\left\langle\frac{v^{2}}{\tau_{\text {drag }}}\right\rangle,
$$

where $\boldsymbol{v}$ is the velocity vector and the angle brackets denote an area average. If numerical drag dominates, kinetic energy is dissipated by the Shapiro filter, which damps the highest wavenumber components of the flow. Because the highest wavenumber in the GCM is set by the model's grid spacing $\Delta x$, we scale the Shapiro filter's damping timescale as $\tau \sim \Delta x / U$. This means the rate at which numerical drag dissipates kinetic energy is equal to

$$
W_{\text {num }} \sim \frac{U^{2}}{\Delta x / U} \times \frac{p}{g}=\frac{U^{3}}{\Delta x} \times \frac{p}{g} .
$$

Third, we constrain the efficiency $\eta$. Previous work on hurricanes and the atmospheres of rocky planets constrained

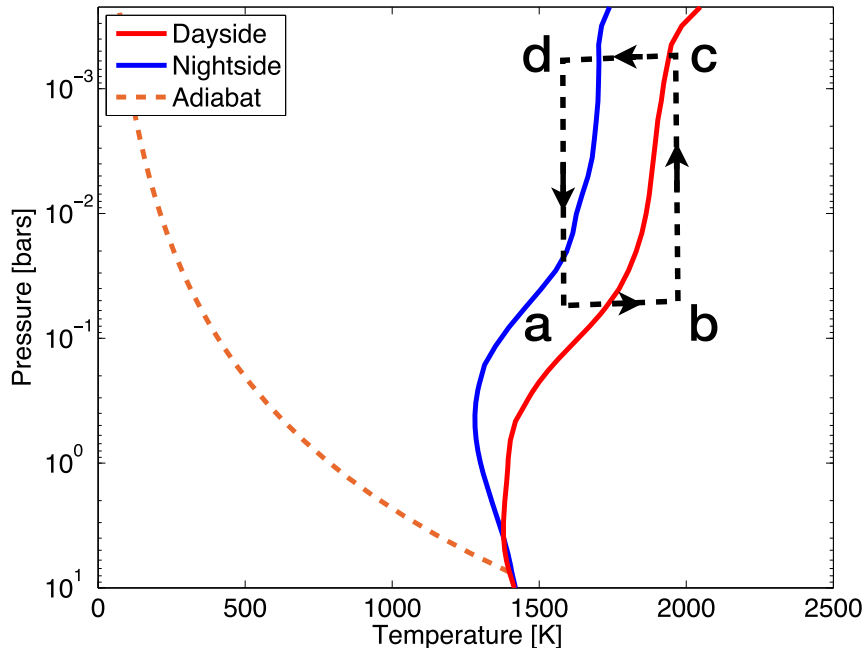

Figure 3. Diagram of the Ericsson cycle, overlaid on dayside- and nightsideaveraged temperature profiles of a reference simulation $\left(T_{\mathrm{eq}}=1500 \mathrm{~K}, \tau_{\mathrm{drag}}=\right.$ $10^{6} \mathrm{~s}$ ) and an adiabatic profile. The Ericsson cycle works as follows: a parcel of fluid starts at depth on the nightside (a), moves toward the dayside (b), where it rises (c), moves back toward the nightside (d), and sinks (a). We assume that rising and sinking motions $(\mathrm{b}-\mathrm{c}, \mathrm{d}-\mathrm{a})$ are isothermal and that motions between hemispheres $(\mathrm{a}-\mathrm{b}, \mathrm{c}-\mathrm{d})$ are isobaric. The isothermal assumption is motivated by the GCM profiles, which show that hot Jupiters are much closer to vertically isothermal than to adiabatic.

this quantity by modeling atmospheric circulations as Carnot cycles (Emanuel 1986; Koll \& Abbot 2016). Unfortunately, it is difficult to argue that hot Jupiters should also resemble Carnot cycles. In a Carnot cycle, parcels of fluid expand and contract adiabatically between heating and cooling. This model is physically motivated by the fact that hurricanes and rocky planets undergo convection, so fluid parcels move rapidly and quasi-adiabatically. In contrast, the upper atmospheres of hot Jupiters are strongly irradiated by their host stars. The irradiation creates a stable stratification and suppresses convection, which means the vertical temperature structure is approximately in radiative equilibrium and lapse rates are small (Iro et al. 2005; Guillot 2010). As the temperature profiles from a reference simulation in Figure 3 illustrate, temperatures are indeed far from adiabatic, which underlines that the Carnot cycle is a poor model for hot Jupiters.

Here we constrain the efficiency $\eta$ by modeling hot Jupiters as Ericsson cycles (McCulloh 1876). The Ericsson cycle is shown in Figure 3: a parcel of fluid starts deep in the nightside atmosphere (Figure 3, point a). It moves at constant pressure toward the dayside (b), where the stellar heating causes it to rise (c). The parcel then moves to the nightside (d), before cooling and sinking back to its starting position (a). Even though the assumption of isothermal vertical motions is an idealization, Figure 3 shows that the Ericsson cycle provides a physically motivated model for hot Jupiters.

The efficiency of the Ericsson cycle is given by

$$
\eta=\frac{\oint \delta Q}{\int_{a}^{c} \delta Q}=\frac{\oint T d s}{\int_{a}^{c} T d s} .
$$

Here $\delta Q$ is a change in a parcel's heat content, and $d s$ is a change in entropy. From the first law of thermodynamics,

$$
T d s=c_{p} d T-\frac{d p}{\rho}=c_{p} d T-R T d \ln p,
$$


where we have used the ideal gas law in the second step. We can then evaluate the numerator $\oint T d s$ as

$$
\begin{aligned}
& \int_{a}^{b} c_{p} d T-\int_{b}^{c} R T d \ln p+\int_{c}^{d} c_{p} d T-\int_{d}^{a} R T d \ln p, \\
= & c_{p}\left(T_{\text {day }}-T_{\text {night }}\right)-R T_{\text {day }} \ln \left(p_{\mathrm{lo}} / p_{\text {hi }}\right) \\
& +c_{p}\left(T_{\text {night }}-T_{\text {day }}\right)-R T_{\text {night }} \ln \left(p_{\mathrm{hi}} / p_{\mathrm{lo}}\right), \\
= & R\left(T_{\text {day }}-T_{\text {night }}\right) \ln \left(p_{\text {hi }} / p_{\text {lo }}\right) .
\end{aligned}
$$

Similarly, the denominator $\int_{a}^{c} T d s$ in Equation (6) is

$$
\begin{aligned}
& \int_{a}^{b} c_{p} d T-\int_{b}^{c} R T d \ln p, \\
= & c_{p}\left(T_{\mathrm{day}}-T_{\mathrm{night}}\right)+R T_{\mathrm{day}} \ln \left(p_{\mathrm{hi}} / p_{\mathrm{lo}}\right) .
\end{aligned}
$$

The ratio of these two terms gives the efficiency, which we write as

$$
\eta=\frac{\frac{T_{\mathrm{day}}-T_{\text {night }}}{T_{\mathrm{day}}} \times \ln \left[\left(p_{\mathrm{hi}} / p_{\mathrm{lo}}\right)^{R / c_{p}}\right]}{\frac{T_{\mathrm{day}}-T_{\text {night }}}{T_{\mathrm{day}}}+\ln \left[\left(p_{\mathrm{hi}} / p_{\mathrm{lo}}\right)^{R / c_{p}}\right]} .
$$

Importantly, the efficiency $\eta$ is always lower than the efficiency of a Carnot cycle, $\eta_{\text {Carnot }}=\left(T_{\text {day }}-T_{\text {night }}\right) / T_{\text {day }}$, which is the maximum efficiency a heat engine can reach. The lower efficiency arises because heat is radiated to space as a parcel passes from the dayside to the nightside (c-d). If, instead, this heat could be stored and used later to heat up the parcel as it passes back from the nightside to the dayside $(\mathrm{a}-\mathrm{b})$, the Ericsson cycle's efficiency would equal that of a Carnot cycle. $^{4}$

As an example, we consider the efficiency of WASP-18b, whose phase curve is consistent with zero heat redistribution from dayside to nightside (Maxted et al. 2013). We assume that a parcel of fluid moves two scale heights in the vertical every time it traverses the planet horizontally, ${ }^{5}$ so $\ln \left[\left(p_{\mathrm{hi}} / p_{\mathrm{lo}}\right)^{R / c_{p}}\right] \sim 2 R / c_{p}$. In this case, WASP-18b's Carnot efficiency would be unity, $\eta_{\text {Carnot }}=1$, whereas its actual efficiency is smaller by a factor of three, $\eta=0.36$. Hot Jupiters can therefore be thought of as comparable to, but less efficient than, ideal Carnot engines. Their efficiency can be reduced even further by molecular diffusion and irreversible phase changes (Pauluis \& Held 2002), so Equation (10) should be considered an upper limit.

We are now able to test the extent to which hot Jupiters resemble heat engines. A key prediction of our theory is that wind speeds are sensitive to whether winds are damped by Rayleigh drag or numerical drag. Based on Equations (2)-(5), we expect that winds should scale as the square root of the modified heat input for Rayleigh drag, $U \propto\left(\tau_{\text {drag }} \eta \sigma T_{\text {eq }}^{4}\right)^{1 / 2}$, whereas they should scale as the one-third power of the heat input for numerical drag, $U \propto\left(\Delta x \eta \sigma T_{\mathrm{eq}}^{4}\right)^{1 / 3}$. To compare both

\footnotetext{
4 If the heat lost during (c-d) could be captured and used to heat the parcel during (a-b), then Equation (9) becomes $\int_{a}^{c} \delta T d s=\int_{b}^{c} \delta T d s=R T_{\text {day }} \ln \left(p_{\mathrm{hi}} / p_{\mathrm{lo}}\right)$ and Equation (10) becomes $\eta=\left(T_{\text {day }}-T_{\text {night }}\right) / T_{\text {day }}$.

5 A parcel travels a vertical distance $d_{\mathrm{z}} \sim \frac{W a}{U}$, where $a$ is the planet radius and $W$ is the vertical wind speed. Using characteristic values from a simulation with $T_{\mathrm{eq}}=1500 \mathrm{~K}$ and no drag, $W \sim 10 \mathrm{~ms}^{-1}, U \sim 10^{3} \mathrm{~ms}^{-1}$, and $a=a_{\mathrm{HD} 209458 \mathrm{~b}}$, we find $d_{\text {vert }} \sim 2 H$, where $H$ is the scale height. In agreement with this estimate, we mapped streamfunctions in our simulations and found that the vertical extent of both zonal and meridional flows is normally confined to $\sim 1-3$ scale heights.
}

scalings in a single plot and because $\Delta x$ depends on numerical parameters, we first use the quantity $\tau_{\text {drag }} \eta \sigma T_{\text {eq }}^{4}$.

Figure 4(a) shows that our simulations indeed exhibit a dichotomy between Rayleigh and numerical drag. The $x$-axis shows the scaled heat input $\tau_{\text {drag }} \eta \sigma T_{\text {eq }}^{4}$, while the $y$-axis shows the root-mean-square wind speed, $U_{\mathrm{rms}}=\left(p^{-1} \int\left\langle u^{2}+v^{2}\right\rangle d p\right)^{1 / 2}$, where $u$ and $v$ are the zonal and meridional wind speeds and where we average horizontally and over the meteorologically active region above $p=1$ bar (see Figure 1). To evaluate $\eta$, we use the dayside and nightside brightness temperatures that would be seen by an observer and assume that a parcel crosses two scale heights, $\ln \left[\left(p_{\mathrm{hi}} / p_{\mathrm{lo}}\right)^{R / c_{p}}\right] \sim 2 R / c_{p}$.

We find that wind speeds in most strongly damped simulations with $\tau_{\text {drag }} \leqslant 10^{5} \mathrm{~s}$ increase according to Rayleigh drag (Figure 4(a)). In contrast, winds in simulations with $\tau_{\text {drag }} \geqslant 10^{6} \mathrm{~s}$ increase more slowly and approximately follow the one-third slope predicted for numerical drag. A notable exception to the Rayleigh scaling is given by the hottest simulations with $\tau_{\text {drag }}=10^{3} \mathrm{~s}$ (yellow dots), in which winds increase with a one-third slope instead. This is due to the relative increase of numerical dissipation in strongly damped simulations. At $\tau_{\text {drag }}=10^{3} \mathrm{~s}$, winds are so weak that Rayleigh drag, which is proportional to wind speed, becomes small relative to numerical drag in parts of the model domain. Similarly, our numerical scaling performs worst for simulations with $\tau_{\text {drag }}=10^{7} \mathrm{~s}$ (purple dots), in which wind speeds flatten out at high $T_{\text {eq }}$ even though the heat input keeps increasing. Given that our theory performs well in the strongly damped limit, deviations from it are likely due to inaccuracies in our numerical scaling, which we discuss below.

We now constrain the wind speeds inside a hot-Jupiter atmosphere. If the atmospheric circulation is primarily balancing Rayleigh drag, then wind speeds should scale as

$$
U_{\text {Rayleigh }}=k_{0}\left(\tau_{\text {drag }} \eta \sigma T_{\mathrm{eq}}^{4} \frac{g}{p}\right)^{1 / 2},
$$

whereas if the circulation is balancing numerical drag, then winds should scale as

$$
U_{\text {num }}=k_{1}\left(\Delta x \eta \sigma T_{\mathrm{eq}}^{4} \frac{g}{p}\right)^{1 / 3} .
$$

Here $k_{0}$ and $k_{1}$ are fitting constants of the order of unity that account for various approximations, in particular, our assumption that temperature profiles are isothermal. We use $k_{0}=0.3$ and $k_{1}=1.1$ to match the simulations at $T_{\mathrm{eq}}=3000 \mathrm{~K}$ with $\tau_{\text {drag }}=10^{4} \mathrm{~s}$ and $\tau_{\text {drag }}=\infty$, respectively. We combine Equations (11) and (12) by demanding that a GCM's work output equals whichever is stronger, Rayleigh or numerical drag, so

$$
U=\min \left(U_{\text {Rayleigh }}, U_{\text {num }}\right) .
$$

To evaluate Equation (12), we use the model's grid spacing at the equator $\Delta x \sim 2 \pi a / 128$, where $a$ is the planetary radius.

We find that our theory matches the GCM simulations well. Figure 4(b) compares our predicted winds with the simulated root-mean-square wind speeds $U_{\text {rms }}$, defined above. As in Figure 4(a), we find that our scaling works best in the strongly damped limit, particularly for the simulations with $\tau_{\text {drag }}=$ $10^{4}-10^{5} \mathrm{~s}$, which our scaling matches to better than $33 \%$. These 

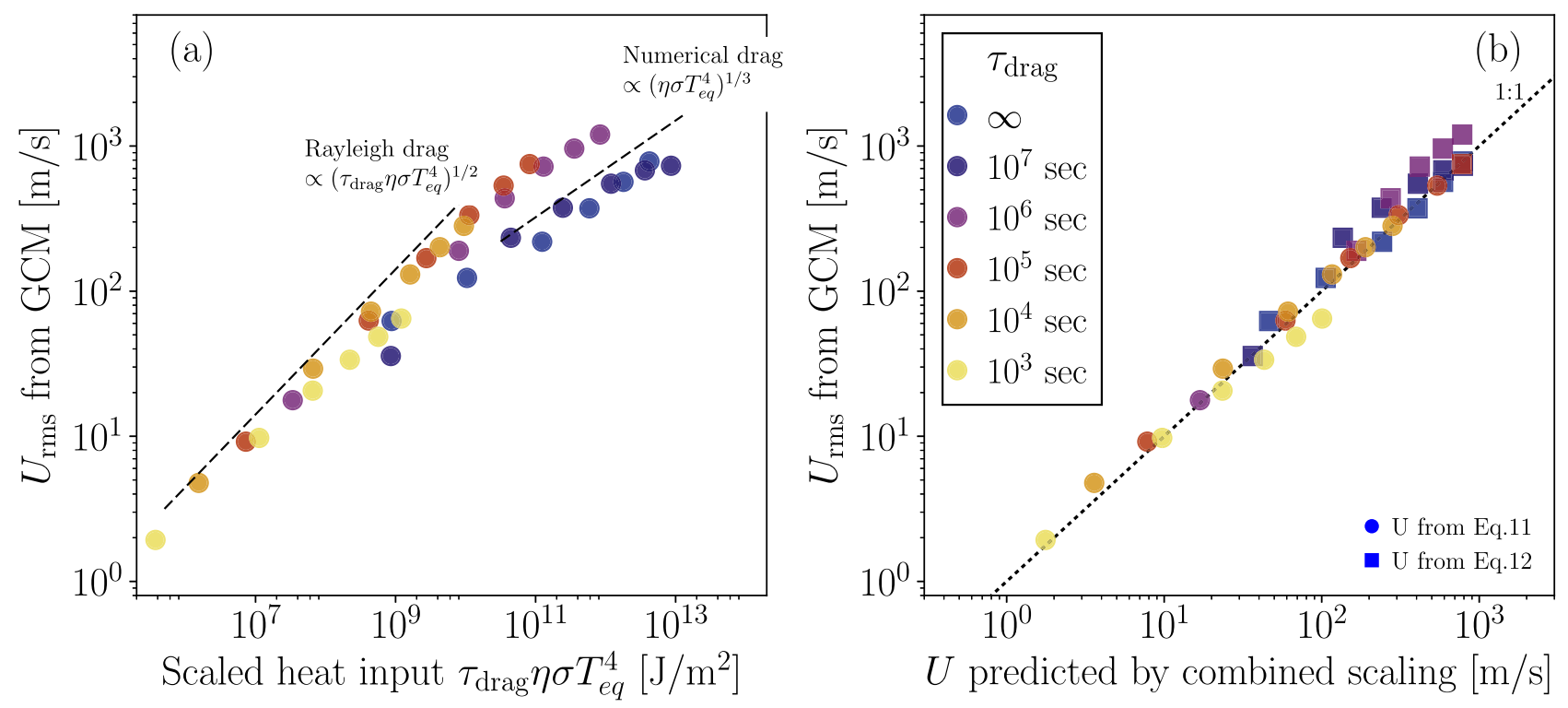

Figure 4. Our heat engine scaling captures the strength of wind speeds across a wide range of hot-Jupiter GCMs. (a) Hot-Jupiter simulations fall into two regimes in which bulk wind speeds either scale following Rayleigh drag or numerical drag (black lines show the two different slopes). (b) Our combined scaling predicts the GCM wind speeds in both regimes. The $y$-axis corresponds to the root-mean-square wind speed averaged over pressures less than 1 bar in different hot-Jupiter simulations, the $x$-axis is the wind speed predicted from Equation (13). Each dot represents a different GCM simulation with varying $T_{\text {eq }}=500-3000 \mathrm{~K}$, where different colors represent different Rayleigh drag timescales used in the simulations. The black line indicates a 1:1 fit between theory and simulations. Circles show simulations for which we use the Rayleigh drag scaling (Equation (11)), squares show simulations for which use the numerical drag scaling (Equation (12)). Note that, to display simulations without Rayleigh drag (blue dots), for which $\tau_{\text {drag }}=\infty$, we use $\tau_{\text {drag }}=5 \times 10^{6} \mathrm{~s}$ in the left panel instead.

are also the simulations in which numerical drag is not yet dominant, and for which we scale winds using Equation (11).

Our scaling additionally matches the weakly damped simulations that are dominated by numerical drag $\left(\tau_{\text {drag }}>10^{5} \mathrm{~s}\right)$, even though the fit is less good than in the strongly damped regime. This is likely due to the approximations we made in deriving Equation (12). To test this point, we performed additional simulations in which we varied the model resolution and timestep. We found that Equation (12) overpredicts the sensitivity of wind speeds to numerical resolution (see the Appendix). Further work is needed to understand exactly how hot-Jupiter simulations equilibrate through numerical drag.

Nevertheless, given that our scaling captures the basic dependence of wind speeds on a planet's heat input (Figure 4(a)) and additionally matches the GCM to better than a factor of two even when the models are dominated by numerical drag (Figure 4(b)), we argue that the main shortcoming in Figure 4 is due to our imperfect description of numerical drag, not due to the heat engine framework. We therefore sidestep the intricacies of numerical simulations and in the last section apply the heat engine framework directly to data.

\section{Evaluating Drag Mechanisms with Observations}

In this section, we use the heat engine framework to predict how strong winds would have to be to balance the two main proposed drag mechanisms on hot Jupiters, namely magnetic drag and shear instabilities. We then evaluate our predictions by comparing them to observed wind speeds obtained from Doppler spectroscopy.

For magnetic drag, we combine Equation (11) with a kinematic scaling for the effective Lorentz drag timescale (Perna et al. 2010; Menou 2012; Rauscher \& Menou 2013). To be consistent with Section 3, we use $k_{0}=0.3$ in Equation (11). The drag timescale is

$$
\tau_{\mathrm{mag}}=\frac{4 \pi H_{e} \rho}{B^{2}}
$$

where $B$ is the dipole field strength, $H_{e}$ is the atmospheric electrical resistivity, and $\rho$ is the gas density. The electrical resistivity is inversely related to the ionization fraction $x_{e}$, $H_{e} \propto \sqrt{T} / x_{e}$, where $x_{e}$ is calculated from the Saha equation (Perna et al. 2010). For hot Jupiters, the ionized gas is largely potassium, for which we assume a solar abundance. ${ }^{6}$ We expect that most dissipation occurs somewhere between the upper levels probed by Doppler observations $\left(\sim 10^{-3}\right.$ bar $)$ and the photosphere, so we calculate winds over the range $10^{-3} \leqslant p \leqslant 1$ bar. Note that Equation (14) does not include induced atmospheric fields. In strongly ionized atmospheres, induced fields can be significant (Rogers \& Showman 2014; Rogers \& Komacek 2014; Rogers \& McElwaine 2017), which means winds could decrease faster with equilibrium temperature than implied by Equation (14).

For shear instabilities, we predict wind speeds analogous to Equation (12). We assume that instabilities have a spatial extent $L$ and damp the flow over a timescale $L / U$, so wind speeds scale as

$$
U_{\text {shear }}=k_{1}\left(L \eta \sigma T_{\mathrm{eq}}^{4} \frac{g}{p}\right)^{1 / 3}
$$

\footnotetext{
6 For a planet with the equilibrium temperature of HD 209458b, $T_{\mathrm{eq}}=1450 \mathrm{~K}$, the ionization fraction is $x_{e}=4.4 \times 10^{-11}$, which is much smaller than the solar abundance of neutral potassium and thus consistent with the approximations made in Perna et al. (2010). The corresponding magnetic resistivity is $H_{e}=2.0 \times 10^{14} \mathrm{~cm}^{2} \mathrm{~s}^{-1}$.
} 

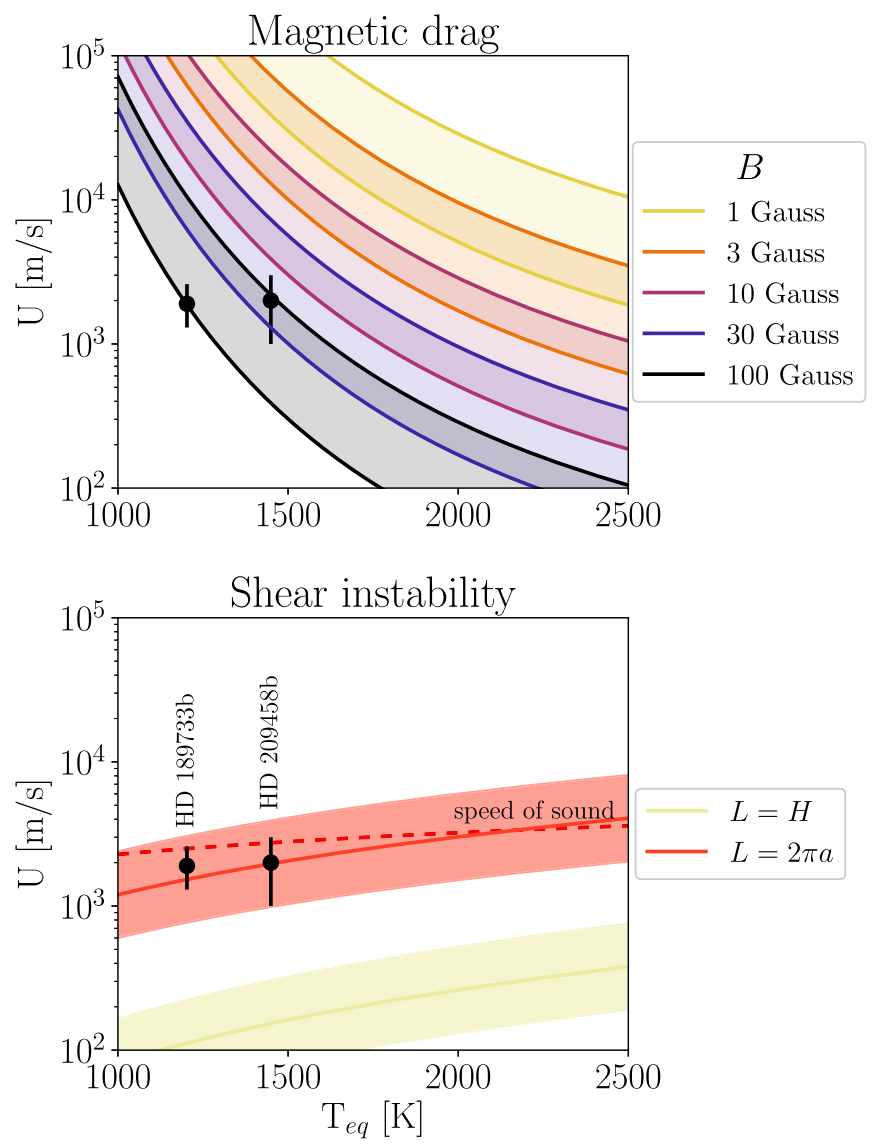

Figure 5. Top: solid lines show the predicted wind speeds from Equation (11), assuming dissipation is caused by magnetic drag. Colored envelopes indicate that our theoretical scalings are subject to uncertainty. The uppermost line for each magnetic field strength shows the wind speed predicted for dissipation occuring at 1 bar, the lower line shows the wind speed predicted for dissipation occuring at $10^{-3}$ bar, and the colored envelope shows intermediate pressures. Dots show wind speeds constrained via Doppler spectroscopy for HD $189733 \mathrm{~b}$ and HD 209458b (Snellen et al. 2010; Louden \& Wheatley 2015). Bottom: solid lines show the predicted wind speeds from Equation (15), assuming dissipation is caused by shear instabilities. Colored envelopes here indicate our estimated uncertainty for our heat engine scaling (see the text). Winds faster than the speed of sound (dashed black line-we assume solar composition and that atmospheric temperature is equal to the equilibrium temperature) can also develop shocks. Magnetic drag can match both observations, but doing so requires a large dipole field $(\gtrsim 100 \mathrm{G})$ for HD 189733b. In contrast, shear instabilities and/or shocks can match the observed wind speeds of both planets.

For consistency, we use $k_{1}=1.1$, as in Section 3. We note that Doppler observations probe the upper atmosphere only whereas our theory constrains large-scale dissipation and thus should be representative of the bulk flow. Observable wind speeds could potentially deviate from the bulk flow in atmospheres with large vertical shear. Nevertheless, we expect that the comparison between our theory and observations is warranted, given that a wide range of hot-Jupiter GCMs produce equatorial jets that are strongly vertically coherent (Showman et al. 2009; Heng et al. 2011a; Liu \& Showman 2013; Mayne et al. 2014; Polichtchouk et al. 2014; Cho et al. 2015).

Figure 5 compares the observed wind speeds of $1.9_{-0.6}^{+0.7} \mathrm{~km} \mathrm{~s}^{-1}$ for HD 189733b (Louden \& Wheatley 2015) and $2 \pm 1 \mathrm{~km} \mathrm{~s}^{-1}$ for HD $209458 \mathrm{~b}^{7}$ (Snellen et al. 2010) with

\footnotetext{
7 Note that these are $1 \sigma$ error bars and the detection itself was only significant at $2 \sigma$.
}

our theoretical predictions for the two drag mechanisms. ${ }^{8}$ To indicate that our scalings are not exact, the colored envelopes in Figure 5 reflect the dominant sources of uncertainty in our scalings. For magnetic drag, the uncertainty is dominated by the pressure at which dissipation is assumed to occur. For shear instabilities, we use the remaining mismatch between theory and GCM simulations ${ }^{9}$ in Section 3. Because the magnetic drag timescale is relatively sensitive to both temperature and pressure, we additionally explored the impact of different pressure-temperature profiles, and find that most features in Figure 5 are robust (see the Appendix).

First, we find that the observations for HD 189733 b can only be matched with a very strong dipole field of $\sim 100 \mathrm{G}$ (Figure 5, top panel). Second, matching the observations for HD 209458b also requires a strong dipole field, on the order of $\gtrsim 30 \mathrm{G}$. Such a dipole is broadly in agreement with predictions from dynamo scaling laws for HD 209458b (Yadav \& Thorngren 2017), which predict a dipole component at the poles of $\sim 50 \mathrm{G}$ (R. Yadav 2017, private communication). We conclude that magnetic drag is a plausible drag mechanism for HD 209458b. In addition, given the potentially large uncertainties in both the Lorentz drag timescale (Equation (14)) and dynamo scaling laws, magnetic drag cannot be ruled out for HD 189733b, even though the required field strengths would be larger than currently expected. Further theoretical work could help reduce these uncertainties. Our result that Lorentz forces are potentially unimportant for HD $189733 \mathrm{~b}$ but may be important for HD 209458b therefore agrees with previous estimates that magnetic drag could become significant at $T_{\text {eq }} \gtrsim 1400 \mathrm{~K}$ (Menou 2012; Rogers \& Komacek 2014).

In contrast to magnetic drag, we find that shear instabilities are a plausible mechanism to match the observations of both planets (Figure 5, bottom panel). Our scaling predicts that wind speeds increase moderately with $T_{\text {eq }}$, in agreement with the observations. We also find that the vertical scale height $H$, which has been proposed as the characteristic scale of KelvinHelmholtz instabilities in hot Jupiters (Goodman 2009; Li \& Goodman 2010), would yield wind speeds that are an order of magnitude too slow to match the observed wind speeds. Instead, a damping length $2 \pi a$, where $a$ is the planet radius, is needed to match the observed wind speeds. Such a damping length could be either due to a horizontal Kelvin-Helmholtz instability or due to the steepening of day-night standing waves into shocks. We note that the shock-resolving simulations in Fromang et al. (2016) also found a dominant scale for horizontal shear instabilities of $L \sim 2 \pi a / 5$, and are thus consistent with our results. The upper end of our wind speed estimate is additionally consistent with the bulk flow becoming supersonic, and thus prone to dissipation via shocks (Figure 5).

\section{Conclusion}

We describe the large-scale atmospheric dynamics of hot Jupiters by modeling them as planetary heat engines. Hot Jupiters are comparable to, but less efficient than, ideal Carnot engines because parcels lose heat to space as they move between dayside and nightside. Our theory successfully captures the intensity of winds in a large number of hot-Jupiter simulations (Figure 4). Remaining differences between theory

\footnotetext{
8 We assume $p=1$ bar, $\eta=0.2$, and $g=23 \mathrm{~m} \mathrm{~s}^{-1}$, with the last two values motivated by the phase-curve amplitude and mass-radius measurements of HD $189733 b$.

9 We conservatively use $100 \%$ uncertainty (a factor of two) for winds predicted with Equation (15).
} 
and simulations are likely due to our imperfect understanding of numerical dissipation in the simulations, instead of a fundamental shortcoming in our theory.

Applying our theory to observations, we find that either the magnetic dipole field of HD 189733 b could be stronger than current estimates suggest, or that its atmosphere is dissipating kinetic energy via shear instabilities and/or shocks. For HD 209458 b, our results indicate that both drag mechanisms can plausibly match the observations.

Looking toward future observations, we expect that magnetic drag should become dominant on hotter exoplanets with $T_{\text {eq }}>$ $1400 \mathrm{~K}$ (Figure 5). Wind speeds on these planets should follow a different trend with equilibrium temperature than wind speeds in colder atmospheres. As a result, we propose that more Doppler measurements over a wider range of planets could reveal a diversity of drag mechanisms at work in hot-Jupiter atmospheres.

We thank Vivien Parmentier, Dorian Abbot, and Malte Jansen for insightful feedback on an early draft. We also thank the reviewer for helpful comments that significantly improved this manuscript. This work benefited from the Exoplanet Summer Program in the Other Worlds Laboratory (OWL) at the University of California, Santa Cruz, a program funded by the Heising-Simons Foundation. D.D.B.K. was supported by a James McDonnell Foundation postdoctoral fellowship. T.D. K. was supported by a NASA Earth and Space Science fellowship.

\section{Appendix A Sensitivity to Numerical Parameters}

Our scalings suggest that, for simulations that are dominated by numerical drag, large-scale wind speeds should be sensitive to horizontal resolution (Equation (12)). To explore this possibility, we performed additional simulations in which we did not include any Rayleigh drag (including no basal drag) and kept the equilibrium temperature fixed to $1500 \mathrm{~K}$, while varying different numerical parameters in the model. The two parameters we considered are the model's horizontal resolution and its timestep $d t$. Table 1 summarizes the numerical parameter variations for this suite of simulations. The Shapiro filter timescale $\tau_{\text {num }}$ was always kept equal to the timestep.

Figure 6 shows that wind speeds are largely independent of the GCM timestep. We only find a $\lesssim 3 \%$ variation in the rms wind speed, while changing $d t$ (and thus also $\tau_{\text {num }}$ ) over an order of magnitude. Given that Equation (12) predicts that wind speeds should be independent of $d t$, this implies a general agreement between our theory and our GCM results.

In addition, Figure 6 shows that large-scale wind speeds are less sensitive to horizontal resolution than our scaling would suggest. Following Equation (12), wind speeds should scale with resolution as $U \propto N_{x}^{-1 / 3}$, where $N_{x}$ is the number of horizontal grid points. Our GCMs do not follow such a scaling and instead we find that the wind speed is independent of resolution to $\lesssim 10 \%$ over a factor of 4 change in horizontal resolution, going from $\mathrm{C} 16$ to $\mathrm{C} 64$. One potential explanation is that our weakly damped simulations develop a direct turbulent cascade of energy to smaller scales, so that the large-scale kinetic energy of the flow becomes insensitive to the dissipation scale. Another explanation is that hot-Jupiter GCM simulations are prone to developing shocks (see

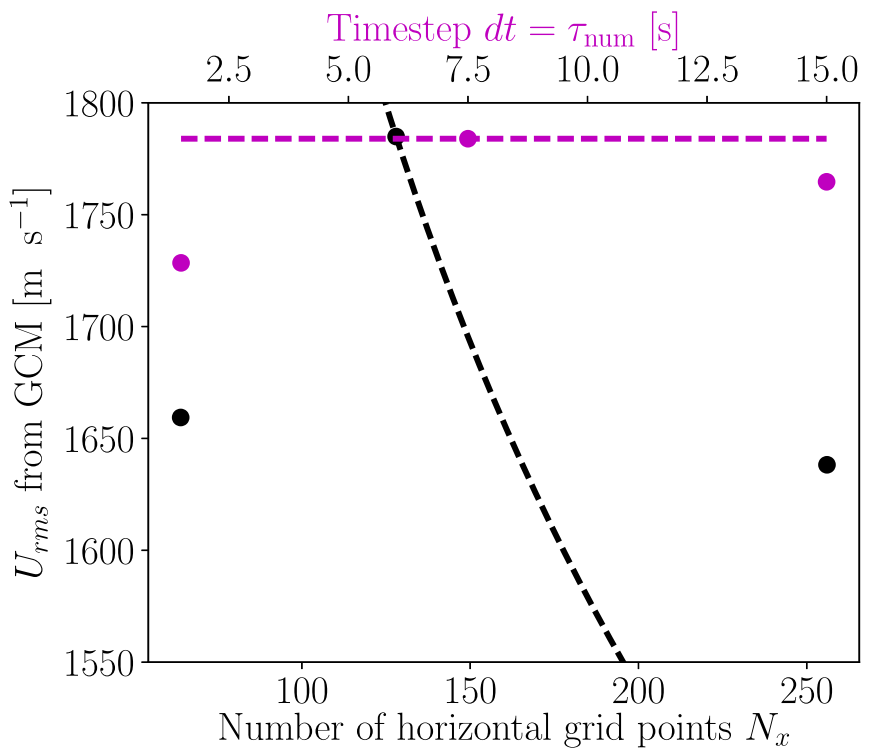

Figure 6. Our scaling for how wind speeds depend on numerical parameters (Equation (12)) matches the independence of $U_{\mathrm{rms}}$ on timestep well, but does not match the dependence of $U_{\mathrm{rms}}$ on grid size. Shown are GCM results for $U_{\text {rms }}$ as a function of horizontal resolution (black dots) and timestep (magenta dots) from simulations with $T_{\mathrm{eq}}=1500 \mathrm{~K}$ and no Rayleigh drag. In this set of simulations, the Shapiro filter timescale $\tau_{\text {num }}$ is kept equal to the timestep. Dashed lines show our predicted dependence of $U_{\text {rms }}$ on timestep (magenta) and resolution (black), using a value of $k_{1}$ such that the theory matches the intermediate GCM point. Equation (12) correctly predicts that the wind speed is independent of timestep (accurate to the $3 \%$ level in our GCMs), but predicts that the wind speeds should decrease steeply with increasing resolution, which is not found in our GCM simulations.

Rauscher \& Menou 2010; Perna et al. 2012; Dobbs-Dixon \& Agol 2013; Fromang et al. 2016), in which case the large-scale kinetic energy might be less sensitive to how well the shock is being resolved than Equation (12) suggests.

Our result is consistent with the suggestion of Heng et al. (2011a) that changes in numerics can change wind speeds in GCMs at the $\lesssim 10 \%$ level, but shows that our scaling does not adequately capture the dependence of large-scale GCM wind speeds on numerical resolution. As a result, a better description of numerical drag than our scaling is needed to capture how hot-Jupiter GCMs converge with numerical drag. Nevertheless, although our numerical scaling overpredicts the sensitivity to numerical parameters, it does correctly predict the sensitivity to physical parameters, such as equilibrium temperature (see Figure 4, left panel).

\section{Appendix B \\ Sensitivity of Magnetic Drag Timescale to Temperature-Pressure Profile}

Because the magnetic drag timescale is highly sensitive to temperature (Perna et al. 2010; Menou 2012; Rauscher \& Menou 2013), we explored the impact of the assumed temperature-pressure profile on our results in Section 4. In Section 4, we assume an isothermal atmosphere, here we constrain the vertical temperature structure using the analytical solutions from Guillot (2010) as follows: we use Equation (29) from Guillot (2010) with parameters similar to those used in that paper $\left(\kappa_{\mathrm{LW}}=10^{-2} \mathrm{~cm}^{2} \mathrm{~g}^{-1}, \gamma=0.1, T_{\mathrm{int}}=100 \mathrm{~K}\right.$, 


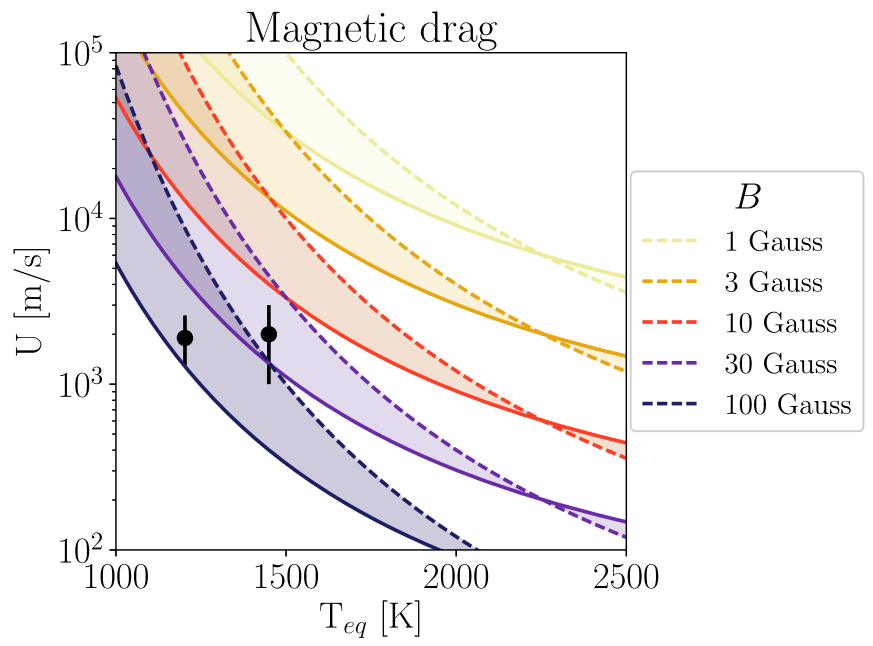

Figure 7. Same as the top panel in Figure 5, but instead of an isothermal atmosphere we assume that temperature increases with pressure following the analytic solutions in Guillot (2010). Solid lines are evaluated at 1 bar, dashed lines are evaluated at $10^{-3}$ bar. Compared with Figure 5, our main conclusions are robust to changes in thermal structure.

$f=0.25)$. With these temperature-pressure profiles, we evaluate the magnetic drag timescale (Equation (14)) at 1 bar and $10^{-3}$ bar, and compute wind speeds following Equation (11).

Figure 7 shows that our conclusions from Section 4 are robust. The most significant difference in Figure 7 compared to Figure 5 occurs above $T_{\text {eq }} \gtrsim 1500 \mathrm{~K}$, in which wind speeds increase more slowly with temperature, whereas our scalings at $T_{\text {eq }}<1500 \mathrm{~K}$ are affected relatively little. The relatively small effect of the temperature-pressure profile is largely due to a trade-off between the effect of pressure and temperature on the magnetic timescale (Equation (14)). Although $H_{e}$ has an exponential sensitivity to temperature, the absolute value of temperature varies less than a factor of two between 1 bar and $10^{-3}$ bar. This compares to a three order of magnitude change in pressure, which appears in both density $(\rho \propto p)$ and resistivity $\left(H_{e} \propto x_{e}^{-1} \propto p^{1 / 2}\right)$ in Equation (14).

\section{ORCID iDs}

Daniel D. B. Koll (ib https://orcid.org/0000-0002-9076-6901 Thaddeus D. Komacek (i) https://orcid.org/0000-00029258-5311

\section{References}

Adcroft, A., Hill, C., Campin, J., Marshall, J., \& Heimbach, P. 2004, MWRv, 132,2845

Brogi, M., de Kok, R., Albrecht, S., et al. 2016, ApJ, 817, 106

Cho, J., Polichtchouk, I., \& Thrastarson, H. 2015, MNRAS, 454, 3423

Crossfield, I., Hansen, B., Harrington, J., et al. 2010, ApJ, 723, 1436

Dobbs-Dixon, I., \& Agol, E. 2013, MNRAS, 435, 3159

Emanuel, K. A. 1986, JAtS, 43, 585

Fromang, S., Leconte, J., \& Heng, K. 2016, A\&A, 591, A144

Goodman, J. 2009, ApJ, 693, 1645

Guillot, T. 2010, A\&A, 520, A27

Heng, K. 2012, ApJL, 761, L1

Heng, K., Frierson, D., \& Phillips, P. 2011a, MNRAS, 418, 2669

Heng, K., Menou, K., \& Phillips, P. 2011b, MNRAS, 413, 2380

Iro, N., Bézard, B., \& Guillot, T. 2005, A\&A, 436, 719

Knutson, H., Charbonneau, D., Allen, L., et al. 2007, Natur, 447, 183

Koll, D., \& Abbot, D. 2015, ApJ, 802, 21

Koll, D., \& Abbot, D. 2016, ApJ, 825, 99

Komacek, T., \& Showman, A. 2016, ApJ, 821, 16

Komacek, T., Showman, A., \& Tan, X. 2017, ApJ, 835, 198

Li, J., \& Goodman, J. 2010, ApJ, 725, 1146

Liu, B., \& Showman, A. 2013, ApJ, 770, 42

Louden, T., \& Wheatley, P. 2015, ApJL, 814, L24

Maxted, P., Anderson, D., Doyle, A., et al. 2013, MNRAS, 428, 2645

Mayne, N., Baraffe, I., Acreman, D., et al. 2014, A\&A, 561, A1

McCulloh, R. 1876, Treatise on the Mechanical theory of Heat and its Applications to the Steam-Engine, etc. (New York: D. Van Nostrand)

Menou, K. 2012, ApJ, 745, 138

Parmentier, V., \& Guillot, T. 2014, A\&A, 562, A133

Parmentier, V., Guillot, T., Fortney, J., \& Marley, M. 2015, A\&A, 475, A35

Pauluis, O., \& Held, I. M. 2002, JAtS, 59, 125

Peixoto, J., \& Oort, A. 1992, Physics of Climate (New York: AIP)

Perna, R., Heng, K., \& Pont, F. 2012, ApJ, 751, 59

Perna, R., Menou, K., \& Rauscher, E. 2010, ApJ, 719, 1421

Polichtchouk, I., Cho, J., Watkins, C., et al. 2014, Icar, 229, 355

Rauscher, E., \& Menou, K. 2010, ApJ, 714, 1334

Rauscher, E., \& Menou, K. 2013, ApJ, 764, 103

Rogers, T., \& Komacek, T. 2014, ApJ, 794, 132

Rogers, T., \& McElwaine, J. 2017, ApJL, 841, L26

Rogers, T., \& Showman, A. 2014, ApJL, 782, L4

Shapiro, R. 1971, JAtS, 28, 523

Showman, A., Fortney, J., Lewis, N., \& Shabram, M. 2013, ApJ, 762, 24

Showman, A., Fortney, J., Lian, Y., et al. 2009, ApJ, 699, 564

Showman, A., \& Guillot, T. 2002, A\&A, 385, 166

Showman, A., Lewis, N., \& Fortney, J. 2015, ApJ, 801, 95

Showman, A., \& Polvani, L. 2011, ApJ, 738, 71

Snellen, I., de Kok, R., de Mooij, E., \& Albrecht, S. 2010, Natur, 465, 1049 Thrastarson, H., \& Cho, J. 2010, ApJ, 716, 144

Tsai, S., Dobbs-Dixon, I., \& Gu, P. 2014, ApJ, 793, 141

Wyttenbach, A., Ehrenreich, D., Lovis, C., Udry, S., \& Pepe, F. 2015, A\&A, 577, A62

Yadav, R., \& Thorngren, D. 2017, ApJL, 849, L12

Zhang, X., \& Showman, A. 2017, ApJ, 836, 73 